\title{
Anesthetic management of Schimke syndrome
}

\author{
Çiğdem Yıldırım Güçlü • Özlem Selvi Can • \\ Menekşe Özçelik
}

Received: 5 August 2013/ Accepted: 6 January 2014/Published online: 29 January 2014

(C) Japanese Society of Anesthesiologists 2014

Keywords Schimke syndrome - General anesthesia . Renal transplantation

To the Editor:

Schimke syndrome is a rare autosomal recessive inherited disorder that is characterized by disproportionately short stature, spondyloepiphyseal dysplasia, hyperlordosis, facial dimorphism resembling mucopolysacchariduria, progressive nephropathy, and defective cellular immunity $[1,2]$. We report the anesthetic management of a 10 -yearold girl with Schimke syndrome undergoing renal transplantation.

It is important to perform a detailed preoperative evaluation in patients with Schimke syndrome. Special attention should be given to airway assessment because of syndrome characteristics such as short neck, microcephaly, lordosis, and facial appearance (ESM Fig. 1). As the patient had a short neck, large tongue, and dimorphic face, we prepared all equipment (different-sized laryngeal mask airways, different-sized laryngoscopes and tracheostomy sets) to be available for the management of a difficult pediatric airway. Although we inserted an appropriate oropharyngeal airway, mask ventilation of the patient was difficult even with two hands, probably because of her large tongue and short neck. After injection of a muscle

Electronic supplementary material The online version of this article (doi:10.1007/s00540-014-1789-7) contains supplementary material, which is available to authorized users.

Ç. Yıldırım Güçlü $(\varangle) \cdot$ Ö. Selvi Can · M. Özçelik

Department of Anesthesiology and ICU, Ankara University

Faculty of Medicine, Sancak mah. 525. Street 15/7,

Çankaya/Ankara, Turkey

e-mail: drcigdemyldrm@yahoo.com.tr relaxant, we could perform mask ventilation and tracheal intubation. We believe that we were able to use the muscle relaxant safely because we had sugammadex for rescue events.

Neurological symptoms in patients with Schimke dysplasia have been described previously as transient ischemic attacks, probably related to vascular stenosis, and ulceration resulting from atherosclerosis and thrombus formation: such stroke episodes can lead to death [3]. Extra care was taken to maintain stable blood pressure during surgery. In conclusion, difficult airway management and special attention to thrombotic events and epileptic seizures are the important issues involved in anesthetic management of this syndrome.

Conflict of interest None.

\section{References}

1. Schimke RN, Horton WA, King CR, Martin NL. Chondtroitin-6sulfate mucopolysaccharidosis in conjuction with lymphopenia, defective cellular immunity and nephrotic syndrome. Birth Defects. 1974;10:258-66.

2. Basiratnia M, Fallahzadeh MH. Schimke immuno-osseous dysplasia. Saudi Med J. 2007;28:457-60.

3. Schmidt B, Christen HJ, Herkenrath P, Benz-Bohm G, MüllerBerghaus J, Querfeld U. Cerebral complications in Schimke immuno-osseous dysplasia. Eur J Pediatr. 1997;156:789-91. 